\title{
Effect of High-and Low-molecular-weight Low-substituted Hydroxyethyl Starch on Blood Coagulation during Acute Normovolemic Hemodilution in Pigs
}

Caroline Thyes, M.D., * Caveh Madjdpour, M.D., ${ }^{\star}$ Philippe Frascarolo, Ph.D., † Thierry Buclin, M.D., $¥$ Marco Bürki, $\$$ Andreas Fisch, Ph.D., || Marc-Alexander Burmeister, M.D.,\# Lars Asmis, M.D., ** Donat R. Spahn, M.D., F.R.C.A.††

Background: Hydroxyethyl starches (HES) with lower impact on blood coagulation but longer intravascular persistence are of clinical interest. The current study aimed to investigate in vivo the isolated effect of molecular weight on blood coagulation during progressive acute normovolemic hemodilution.

Methods: Twenty-four pigs were normovolemically hemodiluted up to a total exchange of $50 \mathrm{ml} \cdot \mathrm{kg}^{-1} \cdot$ body weight $^{-1}$ of HES 650/0.42 or HES 130/0.42. Serial blood sampling was performed to measure HES plasma concentration and to assess blood coagulation. Concentration-effect relations were analyzed by linear regression, followed by the Student $t$ test on regression parameters.

Results: Blood coagulation was increasingly compromised toward hypocoagulability by acute normovolemic hemodilution with both treatments $(P<0.01)$. Significantly greater impact on activated partial thromboplastin time $(P=0.04)$ and significantly stronger decrease of maximal amplitude $(P=$ $0.04)$, angle $\alpha(P=0.02)$, and coagulation index $(P=0.02)$ was seen after acute normovolemic hemodilution with HES 650/0.42 as compared with HES 130/0.42. Except for factor VIII $(P=$ $0.04)$, no significant differences between both treatments were observed when relating antihemostatic effects to HES plasma concentrations $(P>0.05)$. A significantly lesser decrease of hemoglobin concentration has been found with HES 650/0.42 as compared with HES $130 / 0.42(P<0.01)$ in relation to HES plasma concentrations.

Conclusion: High-molecular-weight HES (650/0.42) shows a moderately greater antihemostatic effect than low-molecularweight HES (130/0.42) during acute normovolemic hemodilution. However, similar effects on hemostasis were observed with both treatments when observed antihemostatic effects were related to measured HES plasma concentrations. In addition, HES 650/0.42 may have a lower efficacy in immediately restoring plasma volume.

\footnotetext{
${ }^{*}$ Research Fellow, † Research Associate, †† Professor and Chairman, Department of Anesthesiology, $\ddagger$ Private Docent and Consulting Physician, Division of Clinical Pharmacology and Toxicology, $\S$ Technical Assistant, Department of Experimental Surgery, University Hospital Lausanne. \| Director Pharmaceutical Development, B. Braun Medical SA, Crissier, Switzerland. \# Private docent and Associate Professor of Anesthesiology, Vice President Research and Development, B. Braun Melsungen AG, Melsungen, Germany. ${ }^{* *}$ Head of Coagulation Laboratory, Division of Hematology, University Hospital of Zurich, Switzerland.

Received from the Department of Anesthesiology, University Hospital Lausanne and University of Lausanne, Lausanne, Switzerland. Submitted for publication January 10, 2006. Accepted for publication July 31, 2006. Supported by departmental funds and B. Braun Medical SA, Crissier, Vaud, Switzerland. The funding by B. Braun was exclusively for the experiment and not personal. The publication of the current results did not require a formal authorization of the manufacturer but was discussed with all coauthors, including those being employees of B. Braun (Drs. Fisch and Burmeister). Drs. Burmeister and Fisch are employees of B. Braun AG, Melsungen, Germany, and Crissier, Switzerland, and Dr. Spahn is doing paid consulting for B. Braun Medical SA, Crissier, Switzerland. The Department of Anesthesiology of the University Hospital of Lausanne has done other research projects in collaboration with B. Braun AG, Melsungen, Germany, and Crissier, Switzerland, and has thereby received other funding in the past as well as educational funds from the competitor companies.
}

Address correspondence to Dr. Spahn: Department of Anesthesiology, University Hospital Zurich, Zurich Ch-8091, Switzerland. donat.spahn@usz.ch. Individual article reprints may be purchased through the Journal Web site, www.anesthesiology.org.
HYDROXYETHYL starches (HES) are high-polymeric glucose compounds obtained by hydrolysis and subsequent hydroxyethylation from the waxy maize starch amylopectin. ${ }^{1}$ Mean molecular weight is a major determinant of the physicochemical characteristics of HES preparations. The latter characteristics are furthermore influenced by the molar substitution ratio, which expresses the average number of hydroxyethyl groups added per unit of glucose, and by their $\mathrm{C} 2 / \mathrm{C} 6$ ratio, which refers to the site preference of hydroxyethylation at the glucose subunit carbon atoms occurring mainly at positions $\mathrm{C} 2$ and $\mathrm{C} 6$, and to a lesser degree at position $\mathrm{C} 3{ }^{2}$

Hydroxyethyl starch solutions are considered as effective plasma volume expanders with respect to their colloid oncotic properties and thus are widely used in clinical practice as plasma substitutes. ${ }^{3,4}$ However, the clinical use of HES is limited mainly by their potential to negatively affect hemostasis. Impaired platelet function, ${ }^{5}$ a type I von Willebrand-like syndrome with reduced levels of factor VIII (FVIII) and von Willebrand factor (vWF) exceeding a mere hemodilution effect ${ }^{1,6,7}$ as well as impaired coagulation measured by thromboelastography $\left(\right.$ TEG $^{\circledR}$; Haemoscope Corporation, Niles, IL),${ }^{8,9}$ have been reported. Because of several studies in patients undergoing cardiac surgery showing increased bleeding and transfusion requirements as well as higher mortality after administration of HES 450/0.7 (Hespan; DuPont Pharma, Wilmington, Germany), ${ }^{10-12}$ the US Food and Drug Administration has recently issued a warning against high-volume administration $(>20 \mathrm{ml} / \mathrm{kg}$ ) of the latter HES solution. ${ }^{12}$ Previous studies investigating the antihemostatic effect of different HES solutions have considered molecular weight to be the crucial factor in determining the impact on hemostasis. ${ }^{7,13,14}$ However, this statement was limited by the fact that the reduction of molecular weight of the tested HES solutions has been associated with a concomitant reduction of molar substitution. Although a systematic investigation of the blood coagulation-compromising effect of molecular weight was lacking, the use of HES solutions with lower molecular weight and low molar substitution has been claimed to minimize the risk of coagulopathy. Contrary to the former assumptions, a recent study revealed similar alterations of blood coagulation when high-molecular low-substituted HES was compared with low-molecular low-substituted HES at equal molar substitution. ${ }^{15}$ Therefore, high-molecular low-substituted HES solutions may be expected to show an improved pharmacokinetic 
behavior with a prolonged initial half-life without an exaggerated compromise on blood coagulation. ${ }^{15}$

Currently, the isolated effect of molecular weight on blood coagulation has been examined only after hypervolemic hemodilution and with relatively low doses (20 $\mathrm{ml} \cdot \mathrm{kg}^{-1} \cdot$ body weight $\left.[\mathrm{BW}]^{-1}\right) .^{15}$ Less is known about the effects on in vivo whole blood coagulation after intraoperative colloidal replacement of blood loss with high- and low-molecular-weight low-substituted HES solutions. The aim of this study was to investigate in vivo the blood coagulation-compromising effect of HES solutions of varying molecular weights (HES 650/0.42 vs. HES 130/0.42) at equal molar substitution (0.42) during progressive acute normovolemic hemodilution (up to $50 \mathrm{ml} \cdot \mathrm{kg}^{-1} \cdot \mathrm{BW}^{-1}$ ) in pigs.

\section{Materials and Methods}

The animal experiments were performed according to the guidelines of the Swiss Federal Veterinary Office. The protocol was approved by the Veterinary Office of the Canton of Vaud (Service Vétérinaire, Lausanne, Canton de Vaud, Switzerland). The study group consisted of 24 pigs with a mean $\mathrm{BW}$ of $43 \pm 4 \mathrm{~kg}$. The pigs were fasted overnight but allowed free access to water.

\section{Animal Preparation}

At the time of their arrival at the laboratory, the animals received intramuscular premedication with $0.5 \mathrm{mg} / \mathrm{kg}$ xylazine (Rompun 2\%; Bayer AG, Leverkusen, Germany), 20 $\mathrm{mg} / \mathrm{kg}$ ketamine (Ketaminol 10; Veterinaria AG, Zurich, Switzerland), and $1 \mathrm{mg}$ atropine (Atropinum sulf.; Sintetica S.A., Mendrisio, Switzerland). After the animals were sedated, anesthesia was induced by administration of 3\% halothane (Halothane; Dräger, Lübeck, Germany) by mask, followed by tracheal intubation.

Controlled ventilation was performed using tidal volumes of $10 \mathrm{ml} / \mathrm{kg}$ and a ventilatory rate adjusted to maintain alveolar carbon dioxide pressure at 35-40 mmHg (13-18 $\left.\mathrm{min}^{-1}\right)$. The inspired oxygen fraction was monitored and maintained at 1.0 during surgical instrumentation. Thereafter, ventilation was continued with an inspired oxygen fraction of 0.4 (air- oxygen mixture). Anesthesia was maintained by means of $0.8-2.0 \%$ halothane based on heart rate and blood pressure response to surgical stimulation of each individual animal and left unchanged during the entire study. The ear vein was cannulated (18-gauge cannula). Analgesia was guaranteed by administration of a continuous intravenous infusion of fentanyl (Sintetica S.A., Mendrisio, Switzerland) in a dosage of $4 \mu \mathrm{g} \cdot \mathrm{kg}^{-1} \cdot \mathrm{h}^{-1}$ during the entire period of surgical instrumentation.

A catheter was placed into the internal jugular vein for normovolemic hemodilution, measurement of central venous pressure, and venous blood withdrawal for lab- oratory measurements. A further catheter was placed in the carotid artery, allowing continuous measurement of mean arterial blood pressure and blood withdrawal for arterial blood gas analyses. Via the contralateral carotid artery, an arterial cannula (AS10V; Jostra AG, Hirrlingen, Germany) was inserted and connected to a cardioplegia pump (SIII Double Head Pump; Stöckert, Munich, Germany) for controlled withdrawal of blood for normovolemic hemodilution.

Further monitoring of the animals included continuous three-lead electrocardiogram, heart rate reading, and temperature monitoring. In addition, a direct bladder catheterization was performed for urine sampling.

\section{HES Characterization}

Hydroxyethyl starch solutions with different molecular weights (647 and $136 \mathrm{kd})$ but identical molar substitution (0.42) as well as identical C2/C6 ratio (5:1) were investigated. To ensure full identity of molar substitution and hydroxyethylation pattern, both HES colloids were manufactured using the same raw HES preparation by successive hydrolysis. Thin-boiling waxy maize starch was suspended in water, activated by means of sodium hydroxide, and allowed to react with ethylene oxide for $2 \mathrm{~h}$ at $40^{\circ} \mathrm{C}$. The amounts of waxy maize starch and ethylene oxide were chosen to yield HES with a molar substitution of 0.42 . This original HES with its unique hydroxyethylation pattern was stepwise hydrolyzed thereafter by means of treatment with hydrochlorid acid to yield final HES solutions with molecular weights of $647 \mathrm{kd}$ (HES 650) and $136 \mathrm{kd}$ (HES 130). These were treated with activated carbon, purified by ultrafiltration, diluted to a final concentration of $6 \%$ (wt/vol) in isotonic saline, filled in glass bottles of $500 \mathrm{ml}$ each, and heatsterilized at $121^{\circ} \mathrm{C}$ for $20 \mathrm{~min}$. For determination and verification of HES molecular weights, HES sample solutions were analyzed in duplicate by gel permeation chromatography-multiangle laser light scattering (GPCMALLS; Wyatt Technology, Woldert, Germany) at a flow rate of $1 \mathrm{ml} / \mathrm{min}$ in $70 \mathrm{~mm}$ phosphate buffer, $\mathrm{pH} 7.0$, using serial gel permeation chromatography columns HEMA Bio 40, 100, and 1000 (PSS, Mainz, Germany). Mean average molecular weight was calculated using Astra software (Wyatt Technology). The degree of molar substitution of the HES solution was determined and verified in duplicate according to the method described by Hodges et $a .^{16}$ and Lee et al. ${ }^{17}$

\section{Experimental Protocol}

A total of 24 pigs underwent permuted block randomization for assignment to the study group ( $\mathrm{n}=12$ ), receiving HES 650/0.42, or the control group $(n=12)$, receiving HES 130/0.42. The HES solutions were blinded to the experimental investigators and labeled $\mathrm{K}$ (HES 650/0.42) and L (HES 130/0.42). After all surgical preparations were completed, the animals were allowed to 
recover for $45 \mathrm{~min}$ before the investigational protocol was started. Progressive acute normovolemic hemodilution was induced by steps of $10 \mathrm{ml} \cdot \mathrm{kg}^{-1} \cdot \mathrm{BW}^{-1}$ with a $1: 1$ exchange of blood with either HES 650/0.42 or HES 130/ 0.42 until a total exchange of $50 \mathrm{ml} \cdot \mathrm{kg}^{-1} \cdot \mathrm{BW}^{-1}$ was reached. The HES solution was injected into the right internal jugular vein at the same time and the same rate that blood was removed from the left carotid artery. Every hemodilutional step was conducted over exactly $30 \mathrm{~min}$. At baseline and after each step of $10 \mathrm{ml} \cdot \mathrm{kg}^{-1} \cdot \mathrm{BW}^{-1}$ blood exchange, citrated blood samples were obtained.

\section{Laboratory Measurements}

TEG $^{\circledR}$ Analysis. The TEG ${ }^{\circledR}$ was used to assess the process of blood coagulation by testing the kinetics of the whole coagulation process. ${ }^{18}$ It has been shown previously that $\mathrm{TEG}^{\circledR}$ in conjunction with standard coagulation assays provides a complementary approach suitable for detection of coagulation abnormalities in the course of hemodilution by colloids. ${ }^{7,15}$ Technical details of the $\mathrm{TEG}^{\circledR}$ coagulation analyzer, performance of coagulation tests, and definition of the measured parameters have been described previously. ${ }^{18,19}$

Thromboelastograph analysis was performed using two computerized $\mathrm{TEG}^{\circledR} 5000$ coagulation analyzers. Blood samples were incubated for $1 \mathrm{~h}$ in a $37^{\circ} \mathrm{C}$ water bath ${ }^{18,20}$ followed by blood recalcification and $\mathrm{TEG}^{\circledR}$ analysis according to the manufacturer's instructions. The following TEG $^{\circledR}$ parameters were reported: reaction time (r), coagulation time (k), maximal amplitude (MA), angle $\alpha$, clot firmness (G), and coagulation index. ${ }^{18,20,21}$

Hemoglobin Concentration. Arterial blood samples were collected using heparinized syringes (BD Preset; BD Vacutainer Systems, Plymouth, United Kingdom). Hemoglobin concentration was determined immediately after collection using the Rapidlab 865 analyzer (Bayer Vital GmbH, Fernwald, Germany).

For further laboratory measurements, blood samples were immediately centrifuged at 3,000 rpm for $15 \mathrm{~min}$ at $4^{\circ} \mathrm{C}$ for separation of plasma and blood cellular components (Rotanta/RP; Hettich, Bäch, Switzerland).

Plasmatic Coagulation. Prothrombin time (PT) and activated partial thromboplastin time (aPTT) were determined on an automated coagulation analyzer (BCS; Dade Behring, Marburg, Germany) using a PT reagent containing recombinant tissue factor (Innovin; Dade Behring) and an aPTT reagent containing ellagic acid (Actin FS; Dade Behring), respectively. Functional activity of vWF was determined in a commercial ristocetin-cofactor assay (vWF RCA; Dade Behring) on an automated coagulation analyzer (BCS; Dade Behring). Briefly, vWF activity was assessed by the ability to agglutinate fixed human platelets in the presence of ristocetin. Agglutination was measured turbidimetrically using the coagulation analyzer. FVIII was assessed functionally using factor VIII- deficient plasma (Dade Behring) according to the manufacturer's instructions.

Blood and Plasma Viscosity. Measurement was performed immediately after collection using the plate cone technique at $38^{\circ} \mathrm{C}$ for linear increasing shear rates from 1 to $900 \mathrm{~s}^{-1}$ in $3 \mathrm{~min}$ (Rheostress1; Thermo Haake, Karlsruhe, Germany). Mean viscosities at shear rates from 300 to $900 \mathrm{~s}^{-1}$ were analyzed.

HES Concentration. Hydroxyethyl starch concentration was quantified after extraction from plasma and urine, respectively, and hydrolysis to glucose monomers. Briefly, plasma and urine samples $(1 \mathrm{ml})$ were incubated at $100^{\circ} \mathrm{C}$ for $60 \mathrm{~min}$ after addition of $0.5 \mathrm{ml} \mathrm{KOH}$ solution 35\% (wt/wt) (Fluka, Buchs, Switzerland). HES was precipitated by adding of $10 \mathrm{ml}$ ice-cold absolute ethanol (Fluka) to the supernatant of the reaction mixture and acidly hydrolyzed in $2 \mathrm{~N} \mathrm{HCl}$ (Fluka) for $60 \mathrm{~min}$ at $100^{\circ} \mathrm{C}$. Glucose determination was performed using an enzymatic test kit based on hexokinase/glucose 6-phosphatese (Boehringer Mannheim, Darmstadt, Germany). For determination of HES molecular weight, plasma proteins were eliminated by trichloroacetic acid precipitation (6.4\% [wt/wt] end concentration) and neutralized supernatants were analyzed by GPC-MALLS (Wyatt Technology) at a flow rate of $1 \mathrm{ml} / \mathrm{min}$ in $70 \mathrm{~mm}$ phosphate buffer $\mathrm{pH} 7.0$ using serial GPC columns HEMA Bio 40, 100, and 1000 (PSS).

\section{Data Analysis}

The effects of the high-molecular-weight HES solution (650/0.42) on blood coagulation were compared with those of the low-molecular-weight HES solution (130/0.42) using a two-way analysis of variance for repeated measures (with and without baseline correction) on one way (dilution) with Greenhouse-Geisser correction for assessing solution and dilution effects and their interaction (JMP 5.1 statistical package; SAS Institute, Inc., Cary, NC).

Pharmacokinetic parameters were obtained through individual fitting of the plasma concentration data using the following model of exponential accumulation: Concentration $=$ Css $\cdot(1-\exp (-\lambda \cdot$ time $))($ Css $=$ concentration at steady state; $\lambda=$ accumulation rate constant). This simple model was considered appropriate to fit the study data, which consisted only of isolated concentration values measured before and immediately after each hemodilutional step. The two parameters of the model were expected to be indirectly related with the true pharmacokinetic parameters of the infused products, i.e., Css reflects mainly the clearance and $\lambda$ reflects mainly the half-life. Thus, a clearance-like parameter (CL) could be derived from Css (CL $=$ Dose per dilution unit/Css), and a distribution volume-like parameter (V) could be deduced from $\lambda$ and $\mathrm{CL}(\mathrm{V}=\mathrm{CL} / \lambda)$. In addition, the excretion recovery of the colloids, expressed as a percentage of the cumulated dose infused throughout the experiment, was calculated using the urinary concentration and volume values. Here again, the obtained 
Fig. 1. Effect of progressive acute normovolemic hemodilution with hydroxyethyl starch (HES) 650/0.42 $(n=12)$ and HES $130 / 0.42(n=12)$ on plasma coagulation parameters (prothrombin time [PT; $A]$, activated partial thromboplastin time [aPTT; $B$ ], functional activity of factor VIII [FVIII; $C]$, and functional activity of von willebrand factor [vWF; D]]. PT, aPTT, FVIII activity, and VWF activity were determined before (baseline [BL]) and immediately after each step of acute normovolemic hemodilution $\left(10,20,30,40\right.$, and $50 \mathrm{ml} \cdot \mathrm{kg}^{-1}$ - body weight $\left.{ }^{-1}\right)$. Results are mean \pm SD. Solution effect $\left(P_{\text {sol }}\right)$ and dilution effect $\left(P_{\text {dil }}\right)$ of HES 650/0.42 versus HES 130/0.42 as well as interaction between treatment and dilution $\left(P_{\text {sol }} \times\right.$ dil $)$ as determined by two-way analysis of variance for repeated measurements on one way (dilution).
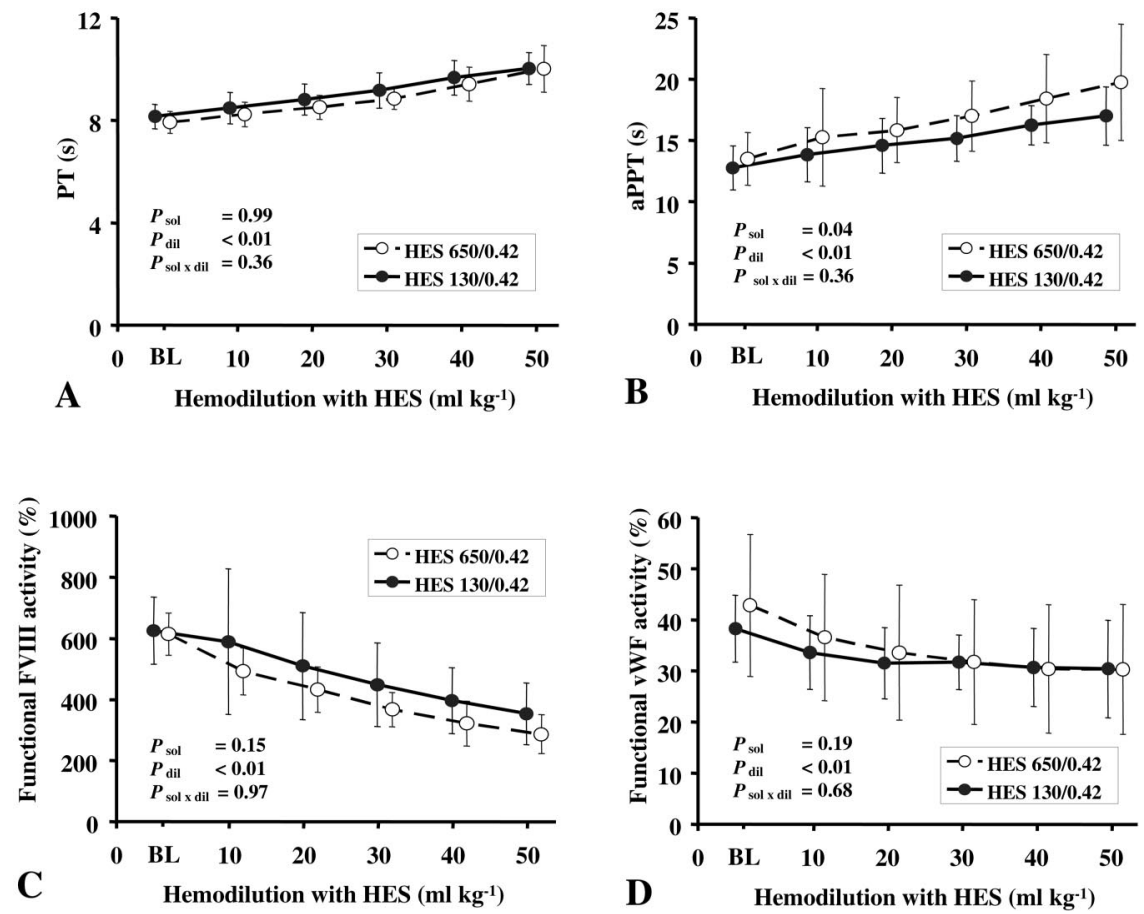

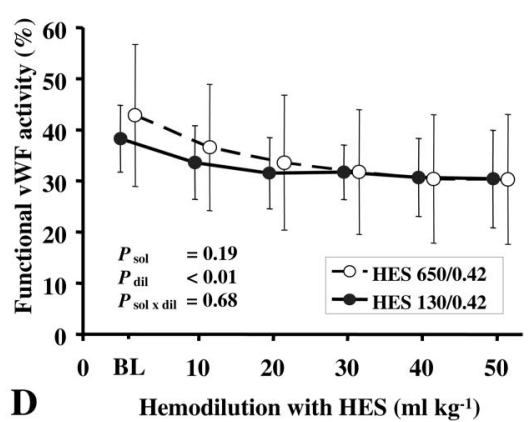

value must be considered as only indirectly related to the true recovery value characterizing the colloids, because the experiment was interrupted before the completion of renal excretion. Because of the approximate model used for fitting, the obtained parameters must not be considered as true pharmacokinetic constants characterizing the colloids. However, under the assumption of roughly similar biases, comparisons between the two colloid solutions tested were possible.

Pharmacodynamic parameters were obtained through the individual fitting of concentration-effect relations, using a simple instantaneous linear model, relating the observed effect to the observed concentration values $\left(\mathrm{C}_{\text {obs }}\right)$ : Thus, for each pharmacodynamic variable, a slope (corresponding to the change of the respective parameter in relation to HES plasma concentration) and an intercept (baseline effect for a zero concentration) were obtained. In a second step, the observed effect was related to the observed HES in vivo molecular weight again; for each pharmacodynamic variable, a slope (corresponding to the change of the respective parameter in relation to HES in vivo molecular weight) and an intercept (baseline effect for a zero HES in vivo molecular weight) were obtained.

Pharmacokinetic and pharmacodynamic parameters obtained from model fitting were thereafter compared between treatments by way of an unpaired Student $t$ test. $P<0.05$ (two-tailed) was considered statistically significant. A sample size determination was performed by a power analysis using data (TEG ${ }^{\circledR}$ parameters MA and angle $\alpha$ ) of a previously published in vivo study. ${ }^{15} \mathrm{~A}$ sample size of 12 animals per group was calculated to ensure $80 \%$ power with an estimated delta of $7.5 \%$ and an SD of $5 \%$. Results are expressed as mean \pm SD.

\section{Results}

No significant differences were found among the HES 650/0.42 and HES 130/0.42 groups with regard to BW and the following variables at baseline: coagulation parameters (TEG ${ }^{\circledR}$ and plasmatic coagulation), hemoglobin concentration, and viscosity $(P>0.05$ for all).

\section{Coagulation Analyses}

Plasmatic Coagulation. Blood coagulation was increasingly compromised by progressive hemodilution with both HES solutions. A significant dilution effect toward hypocoagulability for all plasma coagulation parameters (PT, aPTT, FVIII, and vWF activity) was seen ( $P<0.01$ for all) (figs. 1A-D). On the basis of the baseline-corrected plasma coagulation parameters, HES $650 / 0.42$ showed a significantly greater impact on aPTT compared with HES 130/0.42 ( $P=0.04)$ (fig. $1 \mathrm{~B}$ and table 1$)$, whereas PT $(P=0.99)$, functional FVIII activity $(P=0.15)$, and functional vWF activity $(P=0.19) \mathrm{did}$ not significantly differ from HES 130/0.42 (figs. 1A, C, and $\mathrm{D}$ and table 1 ).

TEG $^{\circledR}$. Except for r, all TEG ${ }^{\circledR}$ parameters showed a significant dilution dependency toward hypocoagulability after infusion of the respective HES solution $(P<$ 0.01 for all) (figs. $2 \mathrm{~A}-\mathrm{F}$ ). On the basis of the baselinecorrected TEG ${ }^{\circledR}$ parameters, a significantly stronger decrease of MA $(P=0.04)$, angle $\alpha(P=0.02)$, and coagulation index $(P=0.02)$ was seen after progressive 
Table 1. Changes of Coagulation Parameters during Acute Normovolemic Hemodilution $(50 \mathrm{ml} / \mathbf{k g}$ )

\begin{tabular}{lcc}
\hline & \multicolumn{2}{c}{$\begin{array}{c}\text { Changes during Acute } \\
\text { Normovolemic Hemodilution }(50 \mathrm{ml} / \mathrm{kg})\end{array}$} \\
\cline { 2 - 3 } & $\begin{array}{c}\text { HES } 650 / 0.42 \\
(\mathrm{n}=12)\end{array}$ & $\begin{array}{c}\text { HES 130/0.42 } \\
(\mathrm{n}=12)\end{array}$ \\
\hline Coagulation parameters & & \\
PT, s & $2.09 \pm 0.75 \dagger$ & $1.88 \pm 0.46$ \\
aPTT, s & $6.25 \pm 2.93^{*}$ & $4.25 \pm 2.60$ \\
FVIII, \% & $-328 \pm 34 \dagger$ & $-272 \pm 102$ \\
vWF, \% & $-11.5 \pm 8.2 \dagger$ & $-7.8 \pm 7.9$ \\
TEG parameters & & \\
r, mm & $0.95 \pm 1.28 \dagger$ & $0.33 \pm 1.86$ \\
k, mm & $0.38 \pm 0.36 \dagger$ & $0.17 \pm 0.23$ \\
MA, mm & $-10.79 \pm 3.65^{\star}$ & $-8.22 \pm 2.98$ \\
angle $\alpha,^{\circ}$ & $-5.94 \pm 1.81^{*}$ & $-2.48 \pm 3.26$ \\
G, dyne/cm ${ }^{2}$ & $-5,738 \pm 2,311 \dagger$ & $-4,635 \pm 2,297$ \\
Coagulation index & $-2.18 \pm 0.85^{*}$ & $-1.35 \pm 1.13$ \\
\hline
\end{tabular}

Data are mean \pm SD.

${ }^{*} P<0.05$ vs. hydroxyethyl starch (HES) $130 / 0.42$. † Not significant $(P>$ $0.05)$ vs. HES $130 / 0.42$.

aPTT = activated partial thromboplastin time; FVIII = functional activity of factor VIII; $\mathrm{G}$ = clot firmness; $\mathrm{k}$ = coagulation time; $\mathrm{MA}=$ maximal amplitude; PT $=$ prothrombin time; $r=$ reaction time; $v W F=$ functional activity of von Willebrand factor. hemodilution with HES 650/0.42 as compared with HES 130/0.42 (figs. 2C, D, and F and table 1). No significant between-group differences were found for $\mathrm{r}(P=0.11)$, $\mathrm{k}(P=0.09)$, and $\mathrm{G}(P=0.28)$ (figs. $2 \mathrm{~A}, \mathrm{~B}$, and $\mathrm{E}$ and table 1).

\section{Hemoglobin Course}

The hemoglobin concentration decreased similarly from $9.1 \pm 0.8 \mathrm{~g} / \mathrm{dl}$ to $4.6 \pm 0.7 \mathrm{~g} / \mathrm{dl}$ (HES 650/0.42) and from $9.0 \pm 0.4 \mathrm{~g} / \mathrm{dl}$ to $4.8 \pm 0.4 \mathrm{~g} / \mathrm{dl}$ (HES 130/0.42) after acute normovolemic hemodilution with the respective solution $(P<0.01)$ (fig. $3 \mathrm{~A})$.

\section{Viscosity}

Hemodilution with both HES solutions significantly decreased blood viscosity $(P<0.01)$. Plasma viscosity shows a downward trend after acute normovolemic hemodilution with the respective solution $(P=0.06)$. However, no significant intergroup differences were seen $(P=0.53$ for blood viscosity and $P=0.90$ for plasma viscosity) (figs. $3 \mathrm{~B}$ and $\mathrm{C}$ ).
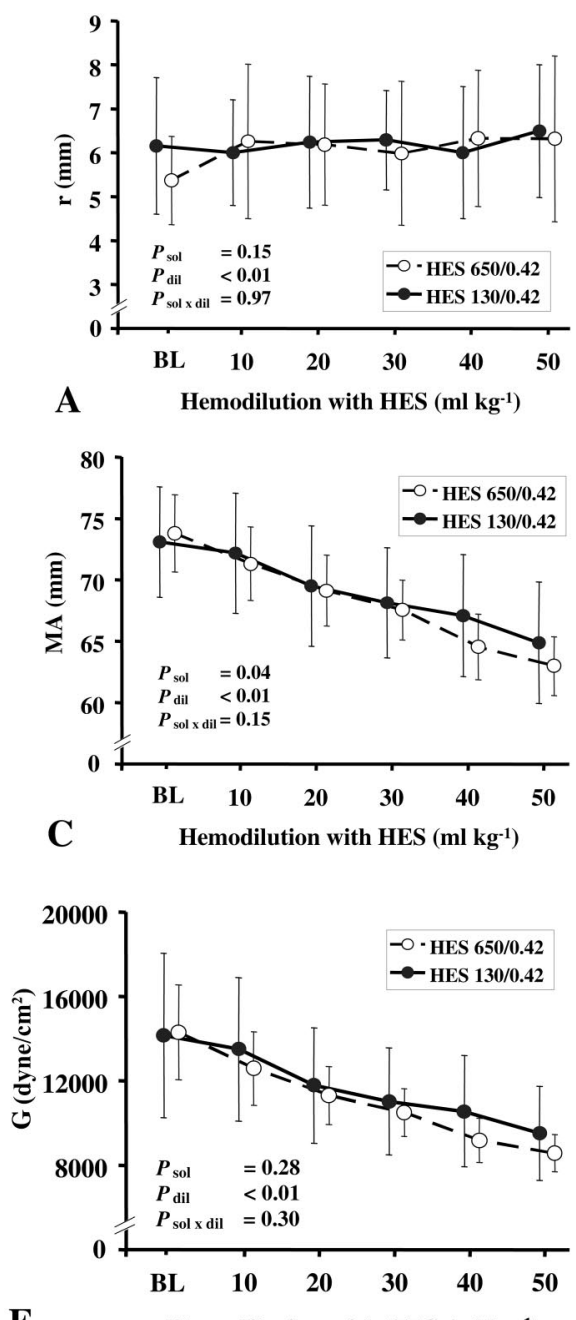

$\mathbf{E}$
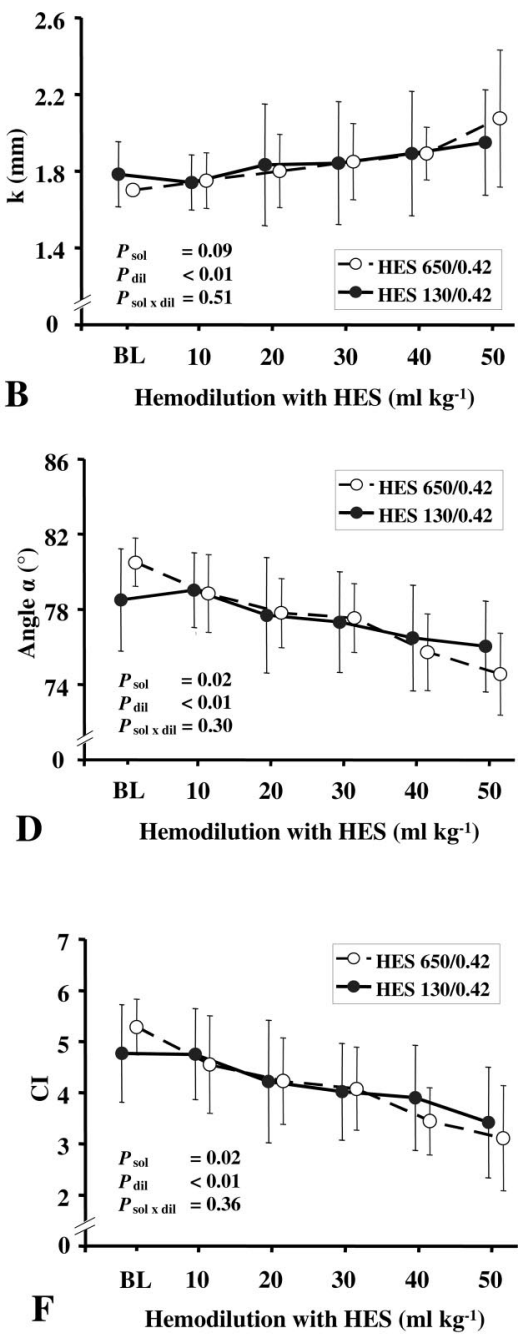

Fig. 2. Effect of progressive acute normovolemic hemodilution with hydroxyethyl starch (HES) 650/0.42 $(n=12)$ and HES $130 / 0.42(n=12)$ on the main TEG $^{\circledR}$ parameters (reaction time $[\mathrm{r} ; A]$, coagulation time $[k ; B]$, maximal amplitude [MA; $C$, angle $\alpha[D]$, clot firmness [G;E], and coagulation index $[\mathrm{CI} ; F])$. TEG ${ }^{\circledR}$ parameters were determined before (baseline [BL]) and immediately after each step of acute normovolemic hemodilution $(10,20,30$, 40 , and $50 \mathrm{ml} \cdot \mathrm{kg}^{-1} \cdot$ body weight $\left.{ }^{-1}\right)$. Results are mean \pm SD. Solution effect $\left(P_{\text {sol }}\right)$ and dilution effect $\left(P_{\text {dil }}\right)$ of HES 650/0.42 versus HES 130/0.42 as well as interaction between treatment and dilution $\left(P_{\text {sol }} \times\right.$ dil $)$ as determined by two-way analysis of variance for repeated measurements on one way (dilution). 


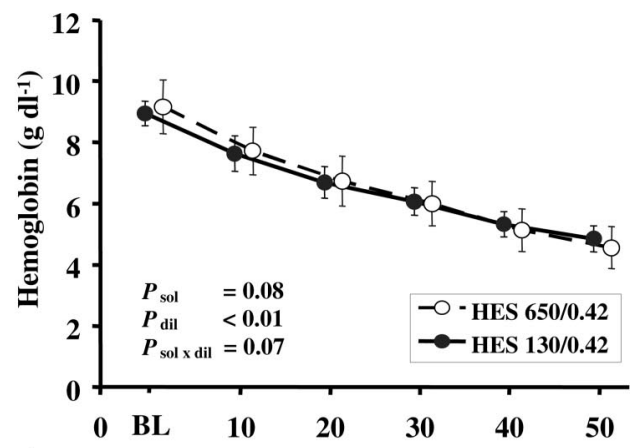

A Hemodilution with HES $\left(\mathrm{ml} \mathrm{kg}^{-1}\right)$
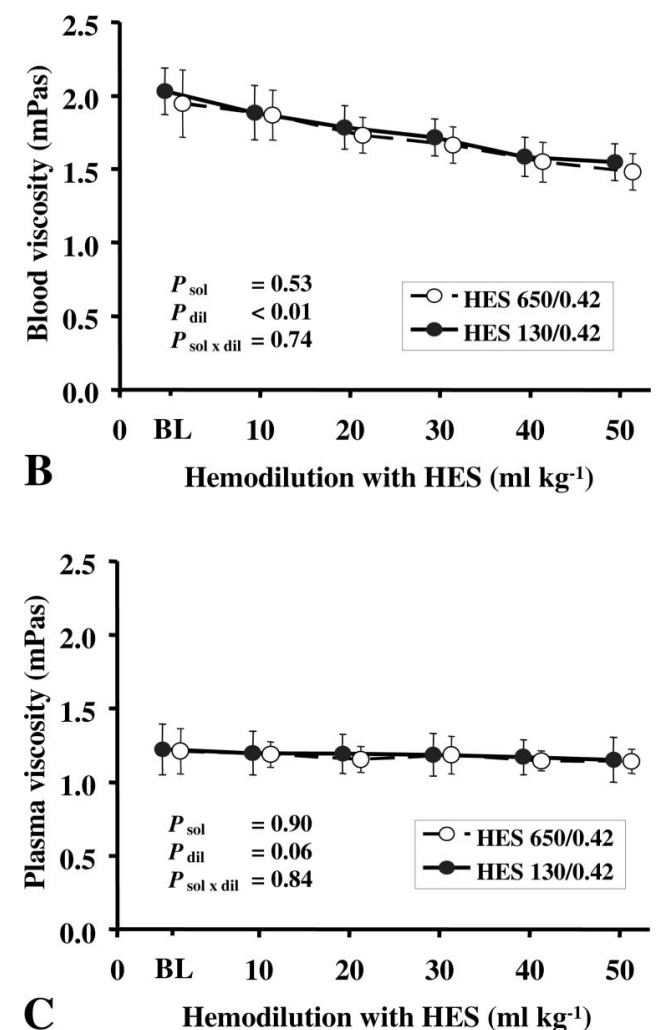

Fig. 3. Effect of progressive acute normovolemic hemodilution with hydroxyethyl starch (HES) 650/0.42 $(n=12)$ and HES $130 / 0.42(n=12)$ on hemoglobin (hemoglobin) concentration $(A)$ as well as mean blood $(B)$ and plasma $(C)$ viscosities at shear rates from 300 to $900 \mathrm{~s}^{-1}$ during progressive acute normovolemic hemodilution. Hemoglobin concentration and mean blood and plasma viscosities were determined before (baseline [BL]) and immediately after each step of acute normovolemic hemodilution $\left(10,20,30,40\right.$, and $50 \mathrm{ml} \cdot \mathrm{kg}^{-1} \cdot$ body weight $\left.{ }^{-1}\right)$. Results are mean \pm SD. Solution effect $\left(P_{\text {sol }}\right)$ and dilution effect $\left(P_{\text {dil }}\right)$ of HES 650/0.42 versus HES 130/0.42 as well as interaction between treatment and dilution $\left(P_{\text {sol }} \times{ }_{\text {dil }}\right)$ as determined by two-way analysis of variance for repeated measurements on one way (dilution).

\section{HES Pharmacokinetics}

Hydroxyethyl starch plasma concentration increased significantly over the whole experimental period after hemodilution with the respective HES solution $(P<0.01$ for both), showing higher plasma concentrations for HES 650/0.42 (9.6 $\pm 1.0 \mathrm{~g} / \mathrm{l})$ as compared with HES 130/0.42
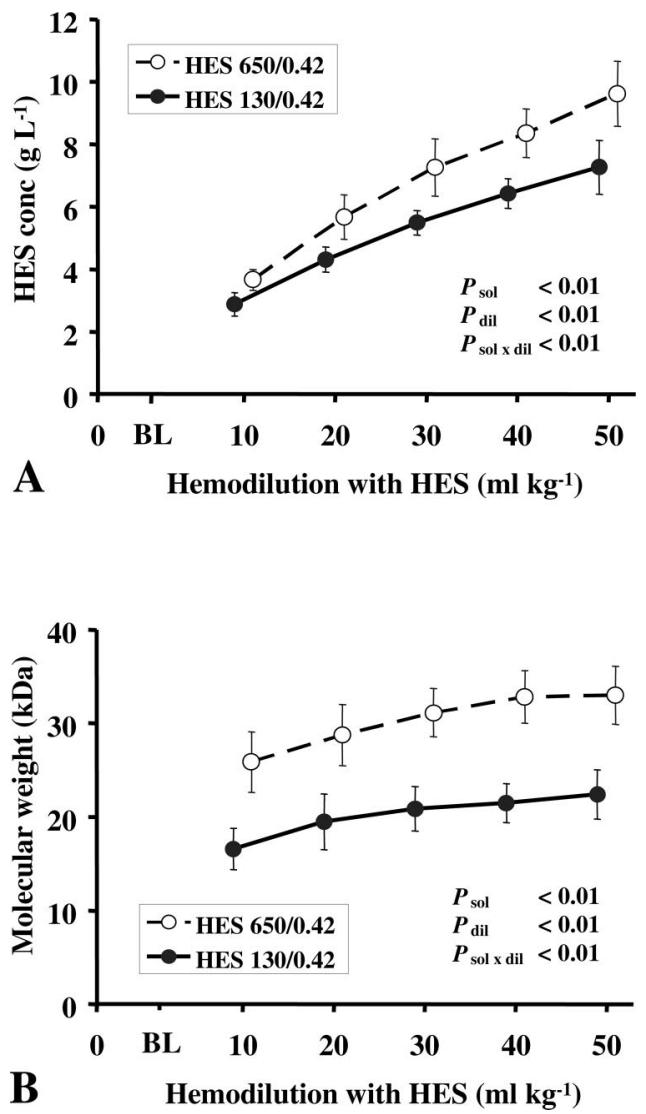

Fig. 4. Effect of progressive acute normovolemic hemodilution with hydroxyethyl starch (HES) 650/0.42 $(n=12)$ and HES $130 / 0.42(n=12)$ on plasma HES concentration in $g / 1(A)$ and as well as molecular weight $(B)$. Plasma HES concentration and molecular weight were determined before (baseline [BL]) and immediately after each step of acute normovolemic hemodilution $\left(10,20,30,40\right.$, and $50 \mathrm{ml} \cdot \mathrm{kg}^{-1} \cdot$ body weight $\left.^{-1}\right)$. Results are mean \pm SD. Solution effect $\left(P_{\text {sol }}\right)$ and dilution effect $\left(P_{\text {dil }}\right)$ of HES $650 / 0.42$ versus HES $130 / 0.42$ as well as interaction between treatment and dilution $\left(P_{\text {sol }} \times\right.$ dil $)$ as determined by two-way analysis of variance for repeated measurements on one way (dilution).

$(7.3 \pm 0.9 \mathrm{~g} / \mathrm{l})(P<0.01)$ (fig. $4 \mathrm{~A})$. Mean in vivo molecular weight increased progressively over the whole experimental period after hemodilution with the respective solution $(P<0.01)$, resulting in higher values for HES $650 / 0.42(32.3 \pm 2.7 \mathrm{kd})$ as compared with HES $130 / 0.42(21.8 \pm 2.5 \mathrm{kd})(P<0.01)$ (fig. 4B). The extrapolated HES concentration at steady state (Css) was significantly higher for HES 650/0.42 (10.2 $\pm 1.2 \mathrm{~g} / \mathrm{l})$ as compared with HES 130/0.42 $(7.7 \pm 1.2 \mathrm{~g} / \mathrm{l})(P<0.01)$ (table 2).

The pharmacokinetic analysis revealed the following treatment effects (table 2): highly significant intergroup differences were found regarding total clearance (CL) and volume of distribution (V), showing smaller values for HES 650/0.42 as compared with HES 130/0.42 $(P<$ 0.01 for all). No significant differences between both treatments appeared regarding the accumulation rate constant $(\lambda)$ and urinary recovery $(P>0.50)$. 
Table 2. Pharmacokinetic Parameters

\begin{tabular}{lcc}
\hline & \multicolumn{2}{c}{ Pharmacokinetic Analysis } \\
\cline { 2 - 3 } & $\begin{array}{c}\text { HES 650/0.42 } \\
(\mathrm{n}=12)\end{array}$ & $\begin{array}{c}\text { HES 130/0.42 } \\
(\mathrm{n}=12)\end{array}$ \\
\hline $\begin{array}{c}\text { Pharmacokinetic parameters } \\
\text { HES concentration at steady } \\
\quad \text { state, g/l }\end{array}$ & $10.2 \pm 1.2^{*}$ & $7.7 \pm 1.2$ \\
$\begin{array}{l}\text { Clearance, l/min } \\
\text { Accumulation rate constant, } \\
\quad \text { min }^{-1}\end{array}$ & $0.084 \pm 0.012^{*}$ & $0.114 \pm 0.020$ \\
$\quad \begin{array}{l}\text { Volume of distribution, I } \\
\text { Urinary recovery, \% }\end{array}$ & $7.39 \pm 1.39^{*}$ & $0.012 \pm 0.003$ \\
\hline
\end{tabular}

Data are mean \pm SD.

${ }^{*} P<0.05$ vs. hydroxyethyl starch (HES) 130/0.42. † Not significant $(P>$ $0.05)$ vs. HES $130 / 0.42$.

\section{Concentration-Effect Analysis}

The concentration curve of the HES solutions was adequately fitted by the instantaneous linear model used in this study as demonstrated by the excellent correlation between observed versus predicted plasma concentrations $\left(r^{2}=0.984\right)$ (fig. $\left.5 \mathrm{~A}\right)$. In addition, the applicability of the model used was confirmed by the absence of
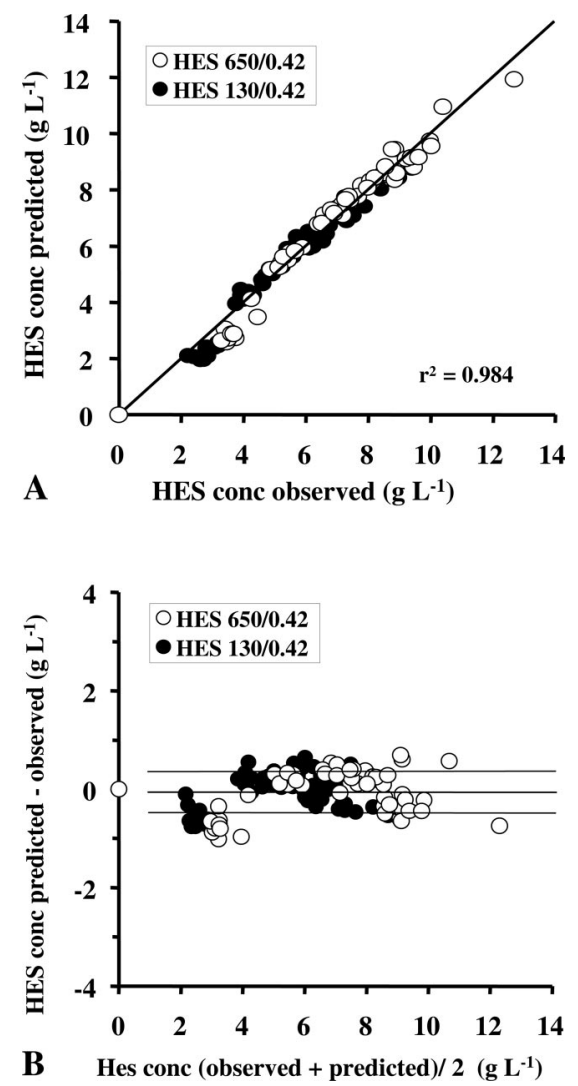

Fig. 5. Pharmacokinetic modeling. The quality of fit between observed hydroxyethyl starch (HES) plasma concentration data (HES conc observed) and predicted data (HES conc predicted) was analyzed by linear regression analysis (HES conc predicted $=$ $-0.107+1.011$ HES conc observed; $\left.r^{2}=0.984\right)(A)$ and BlandAltman analysis (bias $=-0.05 \mathrm{~g} / 1$; precision $[2 \mathrm{SD}]=0.39 \mathrm{~g} / \mathbf{1} ; 95 \%$ confidence interval, -0.44 to $0.34 \mathrm{~g} / \mathrm{l})(B)$.
Table 3. Pharmacodynamic Parameters

\begin{tabular}{lcc}
\hline & \multicolumn{2}{c}{ Changes per g/l Plasma Concentration } \\
\cline { 2 - 3 } & $\begin{array}{c}\text { HES 650/0.42 } \\
(\mathrm{n}=12)\end{array}$ & $\begin{array}{c}\text { HES 130/0.42 } \\
(\mathrm{n}=12)\end{array}$ \\
\hline Pharmacodynamic parameters & & \\
r, mm & $0.08 \pm 0.10 \dagger$ & $0.02 \pm 0.25$ \\
k, mm & $0.03 \pm 0.02 \dagger$ & $0.02 \pm 0.03$ \\
MA, mm & $-1.12 \pm 0.26 \dagger$ & $-1.13 \pm 0.33$ \\
angle $\alpha,^{\circ}$ & $-0.59 \pm 0.17 \dagger$ & $-0.36 \pm 0.42$ \\
G, dyne/cm ${ }^{2}$ & $-598 \pm 190 \dagger$ & $-660 \pm 316$ \\
Coagulation index & $-0.22 \pm 0.05 \dagger$ & $-0.18 \pm 0.14$ \\
PT, s & $0.20 \pm 0.07 \dagger$ & $0.26 \pm 0.06$ \\
aPTT, s & $0.61 \pm 0.21 \dagger$ & $0.57 \pm 0.28$ \\
FVIII, \% & $-34.4 \pm 3.3^{*}$ & $-39.5 \pm 10.8$ \\
vWF, \% & $-1.34 \pm 0.86 \dagger$ & $-1.11 \pm 1.02$ \\
Hb, g/dl & $-0.48 \pm 0.07^{*}$ & $-0.57 \pm 0.06$ \\
\hline
\end{tabular}

Changes of the respective coagulation and $\mathrm{TEG}^{\circledR}$ parameters as well as hemoglobin per g/l plasma concentration of hydroxyethyl starch (HES) 650/ 0.42 and HES 130/0.42, respectively. The concentration-effect relation can be characterized by the extent of the decrease/increase in the respective parameter per $\mathrm{g} / \mathrm{l}$ (i.e., the more negative the number is in the table, the greater is the decrease of the respective parameter per $\mathrm{g} / \mathrm{l}$ plasma concentration of HES; the more positive the number is in the table, the greater is the increase of the respective parameter per $\mathrm{g} / \mathrm{l}$ plasma concentration of HES). Data are mean $\pm \mathrm{SD}$.

${ }^{*} P<0.05$ vs. HES $130 / 0.42$. † Not significant $(P>0.05)$ vs. HES $130 / 0.42$. aPTT = activated partial thromboplastin time; FVIII = functional activity of factor VIII; $\mathrm{G}=$ clot firmness; $\mathrm{Hb}=$ hemoglobin; $\mathrm{k}$ = coagulation time; $\mathrm{MA}=$ maximal amplitude; $P T=$ prothrombin time; $r=$ reaction time; $v W F=$ functional activity of von Willebrand factor.

marked trend across the whole concentration range in the Bland-Altman analysis (fig. 5B).

When relating the observed effect with the measured plasma concentration of HES (table 3), no significant differences between HES 650/0.42 and HES 130/0.42, regarding the $\mathrm{TEG}^{\circledR}$ parameters $(P>0.05$ for all; $\mathrm{r}[P=$ 0.75], k $[P=0.73]$, MA $[P=0.43]$, angle $\alpha[P=0.67]$, $\mathrm{G}[P=0.21]$, and coagulation index $[P=0.71])$ were seen (fig. 6A). Except FVIII $(P=0.04)$, no significant differences with the plasmatic coagulation parameters after comparison of HES 650/0.42 with HES 130/0.42 were found (PT $[P=0.23]$, aPTT $[P=0.79]$, vWF $[P=$ $0.63]$ ) (fig. 6B). The decrease in hemoglobin concentration $(P=0.03)$ (fig. 6C and table 3 ) was less pronounced after progressive hemodilution with HES 650/0.42 as compared with HES 130/0.42.

When relating the observed effect with the measured HES in vivo molecular weights, no significant differences between HES 650/0.42 and HES 130/0.42 regarding the TEG ${ }^{\circledR}$ parameters $(P>0.05$ for all; $\mathrm{r}[P=0.93]$, k $[P=0.63]$, MA $[P=0.69]$, angle $\alpha[P=0.97], \mathrm{G}[P=$ $0.26]$, and coagulation index $[P=0.86]$ ) (fig. 6D) as well as plasmatic coagulation were found $(P>0.05$ for all; PT $[P=0.39]$, aPTT $[P=0.54]$, FVIII $[P=0.17]$, vWF $[P=$ $0.58]$ ) (fig. 6E). The decrease in hemoglobin concentration $(P=0.20)$ (fig. $6 \mathrm{~F}$ ) did not significantly differ after progressive hemodilution with HES 650/0.42 as compared with HES 130/0.42. The consistently higher in 
Fig. 6. Relation of the observed hydroxyethyl starch (HES) plasma concentration values to the observed effects as during acute normovolemic hemodilution with HES 650/0.42 $(\mathrm{n}=12)$ and HES 130/0.42 $(\mathrm{n}=12)$, respectively $(A-C)$. Relation of the measured HES molecular weight to the observed effects during acute normovolemic hemodilution with HES 650/0.42 $(n=12)$ and HES 130/0.42 $(n=12)$, respectively $(D-F) .{ }^{*} P<0.05$ for the slope of HES 650/0.42 versus HES 130/0.42. ns $=$ not significant $(P>0.05)$ for the slope of HES 650/0.42 versus HES 130/ 0.42. aPTT $=$ activated partial thromboplastin time; $\mathrm{CI}=$ coagulation index; $\mathrm{Hb}=$ hemoglobin concentration.
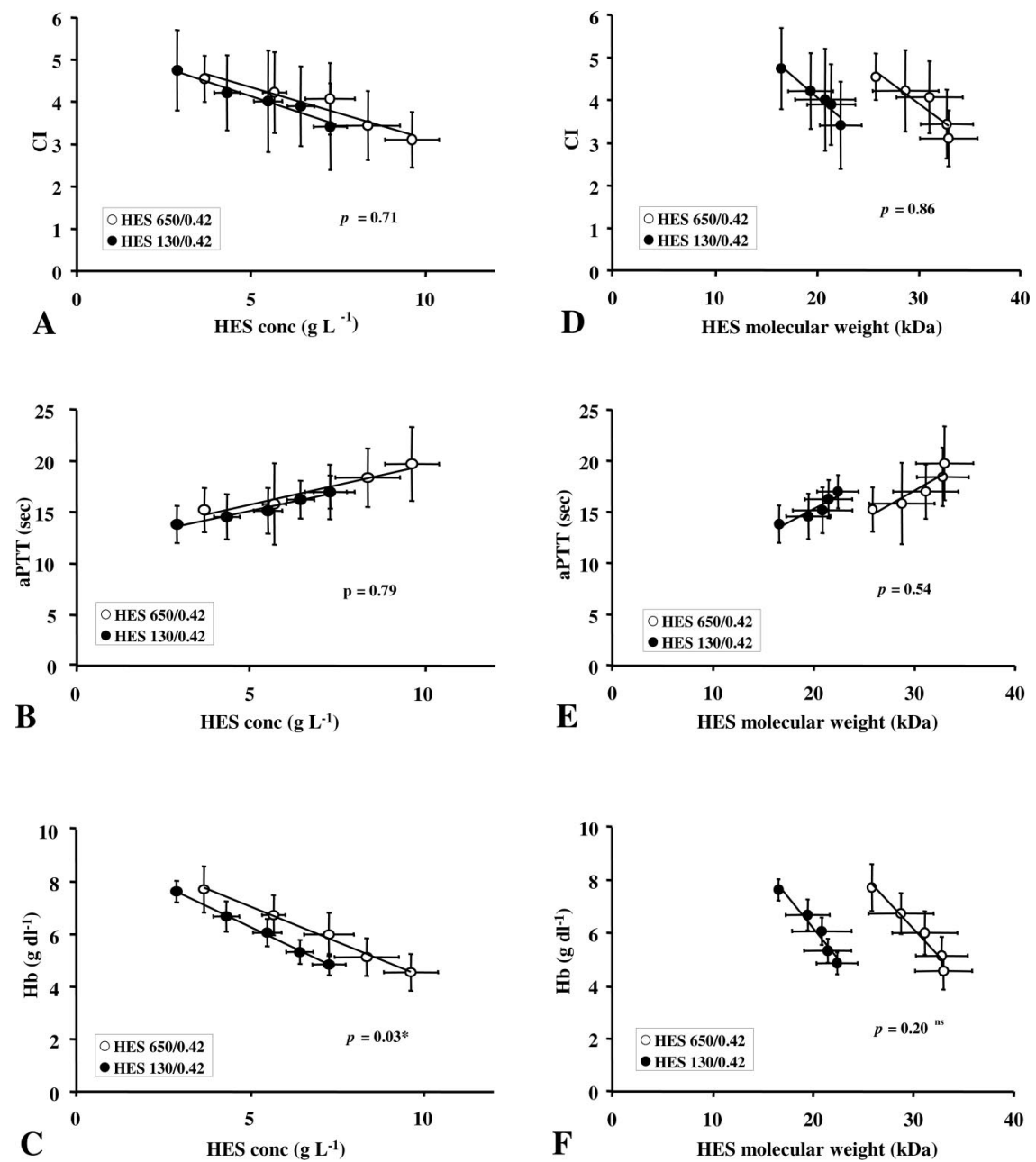

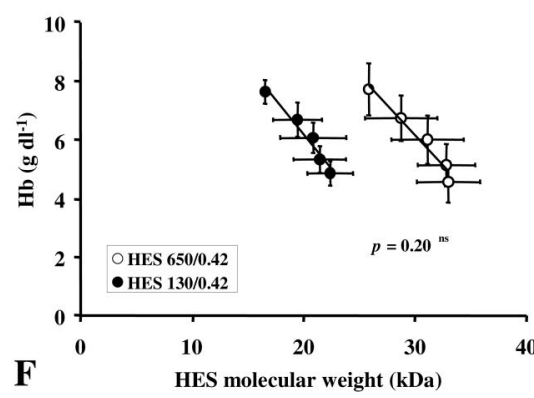

vivo molecular weights during the whole experimental period after hemodilution with HES 650/0.42 (32.3 \pm $2.7 \mathrm{kd})$ as compared with HES 130/0.42 (21.8 $\pm 2.5 \mathrm{kd})$ $(P<0.01)$ (fig. 4B) are translated into the shift of the HES 650/0.42 line to the right (figs. 6D-F).

\section{Discussion}

High-molecular-weight HES (650/0.42) shows a moderately greater antihemostatic effect than low-molecularweight HES (130/0.42) when administered in equal doses during acute normovolemic hemodilution. However, similar effects on hemostasis were observed with both HES solutions when the observed blood coagulation-impairing effects were related to the measured HES plasma concentrations.

Hydroxyethyl starches are polydisperse solutions with a wide variety of physicochemical characteristics in terms of mean molecular weight, degree of substitution, and $\mathrm{C} 2 / \mathrm{C} 6$ ratio accounting for their pharmacokinetic and pharmacodynamic behavior in vivo. ${ }^{1,2,22}$ The interference with blood coagulation as a major adverse effect after HES administration has repeatedly been described and may be regarded as a serious limitation to their clinical use. $^{22}$ Numerous former studies postulated molecular weight being responsible for the observed antihemostatic effects. ${ }^{1,7,9,13,14,23-25}$ However, the main limitation of these studies consisted of a concomitant reduction in molecular weight and molar substitution. Recently, Madjdpour et al $^{15}$ have scrutinized the isolated effect of molecular weight on blood coagulation, showing that low-substituted highmolecular-weight HES (500/0.4 and 900/0.4) influences plasmatic blood coagulation similarly as low-substituted low-molecular-weight HES (130/0.4). Therefore, a high-molecular low-substituted HES solution is expected to show an improved pharmacokinetic behavior with a prolonged initial half-life without an exaggerated compromise on blood coagulation.

Based on these findings, the current study reassessed the blood coagulation-impairing effect of low-substituted high-molecular-weight HES (650/0.42) as compared with low-substituted low-molecular-weight HES (130/0.42) during a clinically more relevant model, i.e., progressive acute normovolemic hemodilution. The impact on hemostasis of the two colloids was analyzed by means of TEG $^{\circledR}$ and plasmatic coagulation tests. 
Most of the $\mathrm{TEG}^{\circledR}$ parameters (figs. 1A-D) as well as all plasmatic coagulation tests (figs. 2A-F) significantly worsened toward hypocoagulability during progressive acute normovolemic hemodilution with the tested solutions. A more pronounced deterioration of TEG ${ }^{\circledR}$ parameters as demonstrated by decreased clot strength (angle $\alpha$ and maximal amplitude) as well as coagulation index has been observed with HES 650/0.42 as compared with HES 130/0.42 (figs. 2C, D, and F and table 1). Regarding plasmatic coagulation tests, the results were less pronounced: Only aPTT was significantly more prolonged with HES 650/0.42 as compared with HES 130/0.42 (fig. $1 \mathrm{~B}$ and table 1). Because progressive normovolemic hemodilution $(50 \mathrm{ml} / \mathrm{kg}$ ) with HES 650/0.42 leads to higher plasma concentrations as compared with HES 130/0.42 (fig. 4A), a subsequent concentration- effect analysis relating the observed effect on blood coagulation with the measured plasma concentration of HES was effected. Except for FVIII, similar alterations of the coagulation parameters after HES 650/0.42 and HES 130/0.42 administration were observed (figs. $6 \mathrm{~A}$ and $\mathrm{B}$ and table 3 ). These results indicate that the blood coagulation-impairing effect of HES solutions is directly governed by the plasma concentration and thus related to the administered volume. In addition, a further analysis relating the observed effects to the measured HES in vivo molecular weights confirmed that molecular weight was not the crucial factor for the observed blood coagulation-compromising effect (figs. 6D and E).

The significantly higher in vivo mean molecular weight of HES 650/0.42 as compared with HES 130/0.42 (fig. 4B) seems to result in a lesser extravasation and slower disappearance rate of HES 650/0.42 (table 2), resulting in higher plasma concentrations of HES 650/ 0.42 as compared with HES 130/0.42 (fig. 4A). Given the volume of distribution and the calculated clearance of both tested HES solutions (table 2), similar elimination half-lives have been found.

Hemoglobin concentration may be used as endogenous point attractor for indirect illustration of the volume effect of infused colloids. Acute normovolemic hemodilution with the respective solution leads to a significant reduction in hemoglobin concentrations $(50 \%$ by HES $650 / 0.42$ and $46 \%$ by HES $130 / 0.42$ ) showing no significant intergroup differences (fig. 3A). When relating the decrease in hemoglobin concentration to the measured plasma concentration of HES, a significantly lesser decrease of hemoglobin concentration has been described with HES 650/0.42 as compared with HES 130/0.42 (fig. 6C and table 3). However, no significant differences after hemodilution with HES 650/0.42 as compared with HES 130/0.42 have been observed by relating the decrease in hemoglobin concentration to the measured HES in vivo molecular weights (fig. 6F). The volume effect of HES solutions depends on the circulating number of osmotically active molecules. ${ }^{2,3}$ The ex- aggerated decrease of the hemoglobin concentration during acute normovolemic hemodilution with HES 130/ 0.42 thus may indicate that more molecules were present than during acute normovolemic hemodilution with HES 650/0.42. This may indeed be the case because the plasma concentration of HES 650/0.42 was only $32 \%$ higher than the plasma concentration of HES 130/0.42 (fig. 4A), whereas the mean molecular weight of HES $650 / 0.42$ was $48 \%$ higher than the mean molecular weight of HES 130/0.42 (fig. 4B). Accordingly, our findings may indicate less effective plasma volume restoration in relation to the plasma concentration of HES 650/0.42 as compared with HES 130/0.42.

Blood rheology has shown to be improved by various mechanisms after HES administration. ${ }^{26,27}$ The predominant rheologic effect of colloids consists in the reduction of whole blood viscosity proportional to the magnitude of their plasma volume expansion. ${ }^{27}$ Plasma viscosity, however, is determined principally by the number and physical properties of dissolved macromolecules. ${ }^{27}$ Accordingly, progressive acute normovolemic hemodilution with HES 650/0.42 and HES 130/0.42, respectively, leads to improved rheologic conditions by demonstrating a significant decrease of whole blood viscosity (24\%) as well as a strong downward trend of plasma viscosity (6\%) (figs. $3 \mathrm{~B}$ and $\mathrm{C}$ ). However, no significant differences between the tested solutions were found.

The pig is a suitable animal model for blood coagulation research. ${ }^{28}$ It has been used to monitor coagulation changes under various circumstances, such as in normovolemic hemodilution, supraceliac aortic cross-clamping, and hemorrhagic shock. ${ }^{29-31}$ Commercially available coagulation tests, functional hemostatic assays, and thromboelastograph analysis (ROTEM; Pentapharm Ltd., Basel, Switzerland) have been shown to be applicable for porcine blood. $^{32,33}$ More importantly, McLoughlin et al. ${ }^{29}$ have shown that the response of PT and aPTT to profound hemodilution is similar in humans and pigs. Porcine coagulation thus reacts quite similarly to hemodilution with HES solutions as compared with the human coagulation system. Nevertheless, precautions must be taken in interpretation of the results as well as in their extrapolation toward human results. ${ }^{33}$ Therefore, in the current study, the known hypercoagulability of the pig may have masked the deleterious effects of both treatments regarding the coagulation parameters, which could have occurred with other species, i.e., humans. Furthermore, no standard platelet aggregation tests have been included in this study, and TEG ${ }^{\circledR}$ may not be adequately sensitive in detecting platelet-related changes in aggregability and function.

Several further limitations of the current study must be considered. Because the current study was not designed for pharmacokinetic analyses, only an approximate pharmacokinetic model was applicable. Therefore, the con- 
clusions drawn by this model involve several assumptions and future studies are necessary to confirm our preliminary findings. In addition, investigation of adverse side effects (i.e., impairment of blood coagulation) of high- and low-molecular-weight low-substituted HES solutions in further clinical settings (acute $v s$. chronic $v$ s. repetitive administration) are desirable to evaluate their clinical utility. Besides the extensively described volume restoring properties, HES seems to exert significant beneficial antiinflammatory effects, ${ }^{34,35}$ such as a diminishment of polymorphonuclear neutrophil sequestration in multiple organs. ${ }^{34,36}$ Recent studies have shown that the clinically relevant effect of HES on leukocyte sequestration depends on the regulation of the integrin function. $^{36}$ The duration of the antiinflammatory effect after HES administration thus may be largely determined by the intravascular elimination of the colloid..$^{36}$ Contrary to these findings, no effect or an apparent proinflammatory action after HES administration has recently be shown by Lang et $a l .{ }^{37}$ Additional studies evaluating the antiinflammatory effect of high-molecular-weight low-substituted HES solutions due to their probable longer intravascular confinement thus seem to be worthwhile.

We conclude that high-molecular-weight HES (650/ 0.42 ) shows a moderately greater antihemostatic effect than low-molecular-weight HES (130/0.42) when administered in equal doses during acute normovolemic hemodilution. However, similar effects on hemostasis were observed with both HES solutions when the observed blood coagulation-impairing effects were related to the measured HES plasma concentrations. In addition, HES 650/0.42 may have a lower efficacy in immediately restoring plasma volume.

\section{References}

1. Treib J, Baron JF, Grauer MT, Strauss RG: An international view of hydroxyethyl starches. Intensive Care Med 1999; 25:258-68

2. Jungheinrich C, Neff TA: Pharmacokinetics of hydroxyethyl starch. Clin Pharmacokinet 2005; 44:681-99

3. Vercueil A, Grocott MP, Mythen MG: Physiology, pharmacology, and rationale for colloid administration for the maintenance of effective hemodynamic stability in critically ill patients. Transfus Med Rev 2005; 19:93-109

4. Boldt J: New light on intravascular volume replacement regimens: What did we learn from the past three years? Anesth Analg 2003; 97:1595-604

5. Deusch E, Gamsjager T, Kress HG Kozek-Langenecker SA: Binding of hydroxyethyl starch molecules to the platelet surface. Anesth Analg 2003; 97:680-3

6. Treib J, Haass A, Pindur G: Coagulation disorders caused by hydroxyethyl starch. Thromb Haemost 1997; 78:974-83

7. Jamnicki M, Bombeli T, Seifert B, Zollinger A, Camenzind V, Pasch T, Spahn DR: Low- and medium-molecular-weight hydroxyethyl starches: Comparison of their effect on blood coagulation. ANESTHESIOLOGY 2000; 93:1231-7

8. Jamnicki M, Zollinger A, Seifert B, Popovic D, Pasch T, Spahn DR: Compromised blood coagulation: An in vitro comparison of hydroxyethyl starch 130/0.4 and hydroxyethyl starch 200/0.5 using thrombelastography. Anesth Analg 1998; 87:989-93

9. Felfernig M, Franz A, Braunlich P, Fohringer C Kozek-Langenecker SA: The effects of hydroxyethyl starch solutions on thromboelastography in preoperative male patients. Acta Anaesthesiol Scand 2003; 47:70-3

10. Knutson JE, Deering JA, Hall FW, Nuttall GA, Schroeder DR, White RD,
Mullany CJ: Does intraoperative hetastarch administration increase blood loss and transfusion requirements after cardiac surgery? Anesth Analg 2000; 90:801-7

11. Sedrakyan A, Gondek K, Paltiel D, Elefteriades JA: Volume expansion with albumin decreases mortality after coronary artery bypass graft surgery. Chest 2003; 123:1853-7

12. Haynes GR, Havidich JE, Payne KJ: Why the Food and Drug Administration changed the warning label for hetastarch. ANESTHESIOLOGY 2004; 101:560-1

13. de Jonge E, Levi M: Effects of different plasma substitutes on blood coagulation: A comparative review. Crit Care Med 2001; 29:1261-7

14. Entholzner EK, Mielke LL, Calatzis AN, Feyh J, Hipp R, Hargasser SR: Coagulation effects of a recently developed hydroxyethyl starch (HES 130/0.4) compared to hydroxyethyl starches with higher molecular weight. Acta Anaesthesiol Scand 2000; 44:1116-21

15. Madjdpour C, Dettori N, Frascarolo P, Burki M, Boll M, Fisch A, Bombeli T, Spahn DR: Molecular weight of hydroxyethyl starch: Is there an effect on blood coagulation and pharmacokinetics? Br J Anaesth 2005; 94:569-76

16. Hodges KL, Kester WE, Wiederrich DL, Grover JA: Determination of alkoxyl substitution in cellulose ethers by zeisel-gas chromatography. Anal Chem $1979 ; 51: 2172-6$

17. Lee YC, Baaske DM, Carter JE: Determination of the molar substitution ratio of hydroxyethyl starches by gas chromatography. Anal Chem 1983; 55: $334-8$

18. Luddington RJ: Thrombelastography/thromboelastometry. Clin Lab Haematol 2005; 27:81-90

19. Chandler WL: The thrombelastography and the thrombelastograph technique. Semin Thromb Hemost 1995; 21:1-6

20. Camenzind V, Bombeli T, Seifert B, Jamnicki M, Popovic D, Pasch T, Spahn DR: Citrate storage affects Thrombelastograph analysis. AnEsthesiology 2000; 92:1242-9

21. Whitten CW, Greilich PE: Thromboelastography: Past, present, and future. ANESTHESIOLOGY 2000; 92:1223-5

22. Kozek-Langenecker SA: Effects of hydroxyethyl starch solutions on hemostasis ANesthesiology 2005; 103:654-60

23. Strauss RG, Pennell BJ, Stump DC: A randomized, blinded trial comparing the hemostatic effects of pentastarch versus hetastarch. Transfusion 2002; 42: $27-36$

24. Langeron O, Doelberg M, Ang ET, Bonnet F, Capdevila X, Coriat P: Voluven, a lower substituted novel hydroxyethyl starch (HES 130/0.4), causes fewer effects on coagulation in major orthopedic surgery than HES 200/0.5. Anesth Analg 2001; 92:855-62

25. Boldt J, Haisch G, Suttner S, Kumle B, Schellhaass A: Effects of a new modified, balanced hydroxyethyl starch preparation (Hextend) on measures of coagulation. Br J Anaesth 2002; 89:722-8

26. Freyburger G, Dubreuil M, Boisseau MR, Janvier G: Rheological properties of commonly used plasma substitutes during preoperative normovolaemic acute haemodilution. Br J Anaesth 1996; 76:519-25

27. Neff TA, Fischler L, Mark M, Stocker R, Reinhart WH: The influence of two different hydroxyethyl starch solutions (6\% HES 130/0.4 and 200/0.5) on blood viscosity. Anesth Analg 2005; 100:1773-80

28. Olsen A, Hansen A, Jespersen J, Marckmann P, Bladbjerg E: The pig as a model in blood coagulation and fibrinolysis research. Scand J Lab Anim Sci 1999; 26:214-24

29. McLoughlin TM, Fontana JL, Alving B, Mongan PD, Bunger R: Profound normovolemic hemodilution: Hemostatic effects in patients and in a porcine model. Anesth Analg 1996; 83:459-65

30. Anagnostopoulos PV, Shepard AD, Pipinos II, Raman SB, Chaudhry PA, Mishima T, Morita H, Suzuki G: Analysis of coagulation changes associated with supraceliac aortic crossclamping using thromboelastography. J Surg Res 2001; 98:52-8

31. Via D, Kaufmann C, Anderson D, Stanton K, Rhee P: Effect of hydroxyethyl starch on coagulopathy in a swine model of hemorrhagic shock resuscitation. J Trauma 2001; 50:1076-82

32. Velik-Salchner C, Schnurer C, Fries D, Mussigang PR, Moser PL, Streif W, Kolbitsch C, Lorenz IH: Normal values for thrombelastography (ROTEM ${ }^{\circledR}$ ) and selected coagulation parameters in porcine blood. Thromb Res 2006; 117:597602

33. Munster A, Olsen A, Bladbjerg E: Usefulness of human coagulation and fibrinolysis assays in domestic pigs. Comp Med 2002; 52:39-43

34. Handrigan MT, Burns AR, Donnachie EM, Bowden RA: Hydroxyethyl starch inhibits neutrophil adhesion and transendothelial migration. Shock 2005; 24:434-9

35. Boldt J: Do plasma substitutes have additional properties beyond correcting volume deficits? Shock 2006; 25:103-16

36. Nohe B, Johannes T, Reutershan J, Rothmund A, Haeberle HA, Ploppa A, Schroeder TH, Dieterich HJ: Synthetic colloids attenuate leukocyte-endothelial interactions by inhibition of integrin function. ANESTHESIOLOGY 2005; 103:759-67

37. Lang JD Jr, Figueroa M, Chumley P, Aslan M, Hurt J, Tarpey MM, Alvarez B, Radi R, Freeman BA: Albumin and hydroxyethyl starch modulate oxidative inflammatory injury to vascular endothelium. ANESTHESIOLOGY 2004; 100:51-8 